\title{
Polyphenols as dietary supplements: A double-edged sword
}

\author{
This article was published in the following Dove Press journal: \\ Nutrition and Dietary Supplements \\ 15 December 2009 \\ Number of times this article has been viewed
}

\section{Keith R Martin \\ Christy L Appel}

Nutrition Program, Healthy Lifestyles Research Center, College of Nursing and Health Innovation, Arizona State University, Mesa, AZ, USA
Correspondence: Keith R Martin Nutrition Program, Health Lifestyles Research Center, College of Nursing and Health Innovation, Arizona State University, 6950 East Williams Field Road Mesa, AZ 8521 2, USA

Tel + I 480 727-1925

Fax + I 480 727-1064

Email keith.r.martin@asu.edu
Abstract: Increased consumption of fruits and vegetables is associated with a lower risk of chronic disease such as cardiovascular disease, some forms of cancer, and neurodegeneration. Pro-oxidant-induced oxidative stress contributes to the pathogenesis of numerous chronic diseases and, as such, dietary antioxidants can quench and/or retard such processes. Dietary polyphenols, ie, phenolic acids and flavonoids, are a primary source of antioxidants for humans and are derived from plants including fruits, vegetables, spices, and herbs. Based on compelling evidence regarding the health effects of polyphenol-rich foods, new dietary supplements and polyphenol-rich foods are being developed for public use. Consumption of such products can increase dietary polyphenol intake and subsequently plasma concentrations beyond expected levels associated with dietary consumption and potentially confer additional health benefits. Furthermore, bioavailability can be modified to further increase absorption and ultimately plasma concentrations of polyphenols. However, the upper limit for plasma concentrations of polyphenols before the elaboration of adverse effects is unknown for many polyphenols. Moreover, a considerable amount of evidence is accumulating which supports the hypothesis that high-dose polyphenols can mechanistically cause adverse effects through pro-oxidative action. Thus, polyphenol-rich dietary supplements can potentially confer additional benefits but high-doses may elicit toxicity thereby establishing a double-edge sword in supplement use.

Keywords: antioxidant, bioavailability, flavonoids, polyphenols, supplement

\section{Introduction}

Increased consumption of fruits and vegetables has been consistently recommended to reduce globally the risk of chronic disease including cardiovascular disease (CVD), cerebrovascular disease, and some forms of cancer. ${ }^{1,2}$ This is particularly relevant to the US, where, collectively, cancer, cardiovascular disease, hypertension, type 2 diabetes, and stroke account for more than $75 \%$ of all deaths. The protection against disease is due, in large part, to the plethora of bioactive molecules, both nutritive and nonnutritive in plants, including the antioxidant polyphenols. ${ }^{3}$ For example, phenolic acids and flavonoids, subclasses of polyphenols, contribute to the distinctive colors of fruits and have been shown to be, in some instances, potently effective against dyslipidemia, CVD, and underlying mechanisms of other disease processes.

Fruits and vegetables contain thousands of biologically active phytochemicals that may exhibit independently or in an interactive manner effects that can prevent disease and promote health, in part, via antioxidant activity. ${ }^{4}$ In fact, research supports prooxidant associated oxidative stress as a contributing factor in $>100$ chronic human diseases. ${ }^{5}$ Given the clear potential for bioactive agents, such as specific polyphenols, submit your manuscript | www.dovepress.com

Dovepress 
to prevent or mitigate the occurrence of chronic disease, there has been considerable interest in developing dietary supplements containing polyphenols and/or polyphenol-rich food products. In such dietary nutrients specific components may be purified and/or concentrated, singly or in combination, to boost the antioxidant status of the consumer. ${ }^{6}$ Furthermore, attempts have been made to increase the bioavailability of dietary polyphenols, specifically absorption, to concentrations higher than commonly associated with dietary sources to ultimately increase plasma and tissue levels. There is however a considerable amount of evidence being accumulated which supports the hypothesis that such compounds, at high concentrations, can cause adverse effects presumably through pro-oxidative effects.

\section{Plant polyphenols}

Plant polyphenols are ubiquitous, essential and located throughout most plant tissues which contribute significantly to plant physiology. They play key roles in pigmentation, growth, reproduction, together with resistance to pathogens and predators, due to their potent astringency and function as phytoalexins. ${ }^{3}$ Plant polyphenols are also extremely important components of the human diet because of their purported antioxidant activity, free radical scavenging abilities and the capacity to mitigate oxidative stress-induced tissue damage associated with chronic disease. Additionally, some polyphenols function as antibiotics and exert antidiarrheal, antiulcer, and anti-inflammatory activities. , $^{3,7}$

In contrast to classic nutrients, ie, vitamins and minerals, plant polyphenols are not required for vital body functions in humans such as growth, reproduction, wound repair, and development, thus they are often referred to as nonnutrients. However, there is compelling clinical and epidemiological evidence that some polyphenols and polyphenol-rich plant sources significantly reduce the risk of chronic diseases. As articulated by Williamson and colleagues, this suggests that polyphenols are nutrient-like and are essential to humans in reaching their full "genetically-determined lifespan," as a result the phrase "lifespan essential" has been coined. ${ }^{9}$ Undoubtedly, dietary polyphenols are beneficial, if not critical, to human health.

Polyphenols, or phenolic compounds, constitute one of most numerous and widely distributed groups of phytochemicals in the plant kingdom. More than 8,000 distinct structures are reportedly known and identified. As molecules, polyphenols are products of secondary metabolism and can vary from simple phenolic acids to highly polymerized molecules such as tannins. Polyphenols exist primarily in a conjugated form with one or more sugar residues, viz., glycosides, linked to one or more of the many hydroxyl groups. Direct links to the sugar ring, which is most commonly glucose, can also occur. Polyphenols without sugar linkages are referred to as aglycones.

As a chemical family, polyphenols can be divided into at least 10 different classes depending on their basic chemical structure (Figure 1). The most common and important low molecular weight phenolic compounds are simple phenolic derivatives and flavonoids. Phenolic acids are simple molecules such as caffeic acid, vanillin, and coumaric acid, that account for approximately one-third of the total dietary intake of polyphenols; flavonoids account for the remaining two-thirds. ${ }^{10}$ To further illustrate the complexity of the groupings, flavonoids can be further subdivided into 13 classes with more than 5,000 described compounds. ${ }^{3,11,12}$

The flavonoids constitute the bulk of naturally occurring dietary polyphenols and, thus, are often the focus of scientific study as one of the most nutritionally important classes of dietary compounds. Flavonoids are found in high concentrations in wine, tea, grapes, cocoa, and a wide variety of plants. ${ }^{13}$ The dietary groupings have been simplified by some to include lignins (nuts, whole grain cereals), proanthocyanidins (grapes, pine bark, cocoa), anthocyanins/ anthocyanidins (brightly colored fruits and vegetables, berries), isoflavones, (soybeans), catechins (tea, grapes, wine), tannins (tea, nuts), quercetin (grapes, wines, onions) and naringenin/hesperidin (citrus fruits). ${ }^{14}$

Many flavonoids occur in plants as aglycones although they are most commonly found as glycoside derivatives. ${ }^{3,12}$ Anthocyanins, glycosides of anthocyanidin, are the most important group of water-soluble plant pigments and contribute to the color of flowers and fruits of higher plants. Most are relatively low molecular weight and soluble, which is dependent in large part on the degree and type of modification of their chemical structure (hydroxylation, glycosylation, acylation). ${ }^{3}$ Chemically, the flavonoids are phenylpropanoid derivatives (C6-C3) consisting of two aromatic rings linked through a three carbon bridge (C6-C3-C6) forming, usually, an oxygenated heterocyclic ring.

While flavonoids are low molecular weight, the tannins are compounds of intermediate to high molecular weight $(\sim 30,000 \mathrm{Da})$ and are more extensively hydroxylated. ${ }^{11}$ These characteristics contribute to the formation of insoluble complexes with carbohydrate and protein conferring the sensation of bitterness or astringency. Overall, the tannins can be subdivided into two major chemical groups to include hydrolysable and condensed tannins. ${ }^{8}$ Hydrolysable tannins are based on a gallic acid monomer and its dimeric condensation 


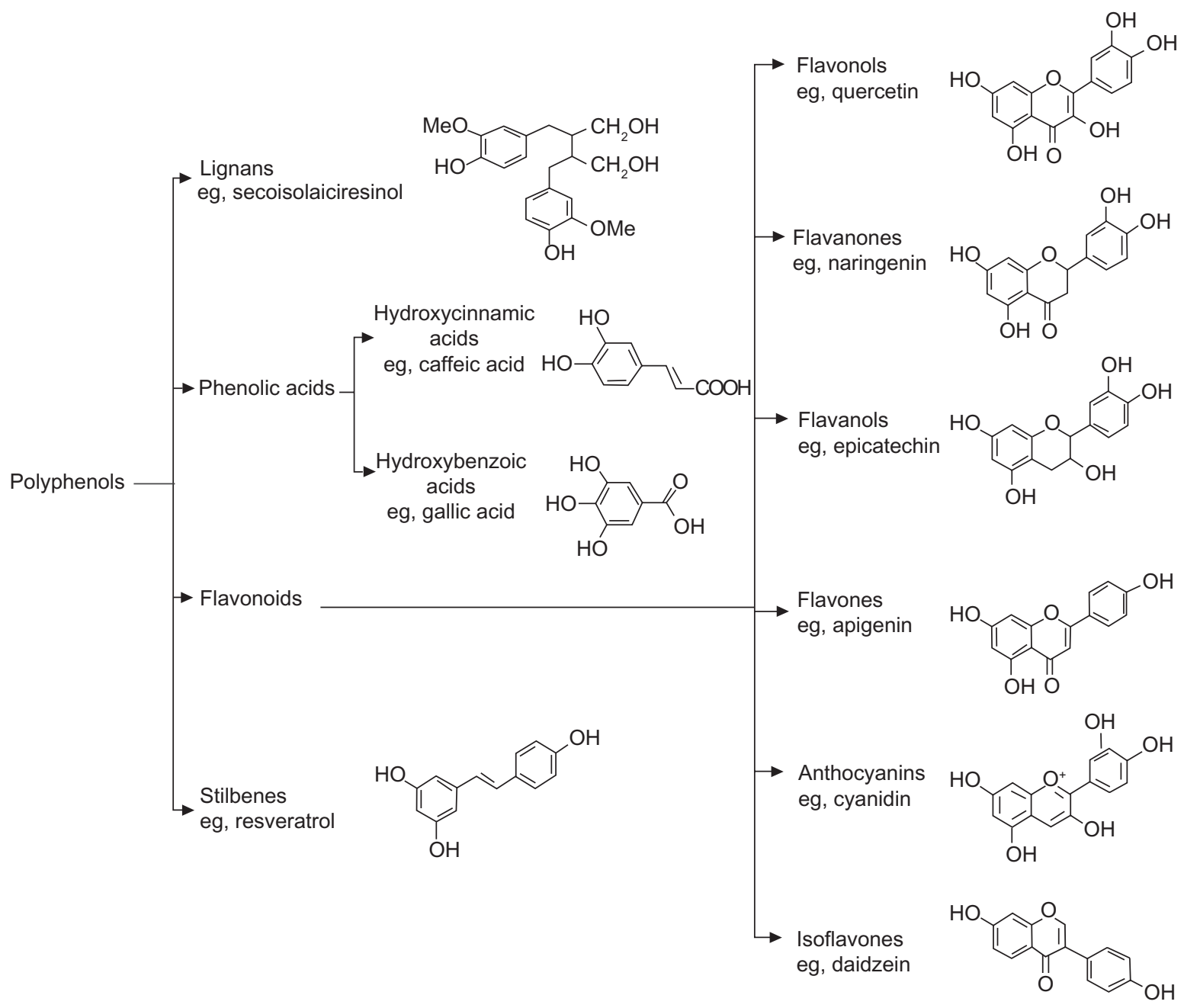

Figure I Classification and chemical structure of major classes of dietary polyphenols. Copyright @ 2008. Reprinted with the permission of Cambridge University Press from Spencer JP, Abd El Mohsen MM, Minihane AM, Mathers JC. Biomarkers of the intake of dietary polyphenols: strengths, limitations and application in nutrition research. Br J Nutr. 2008;99:12-22.

product, hexahydroxydiphenic acid. These are typically esterified to a sugar, which frequently is glucose. It is the interaction, combinations and condensation products of these precursors that generate high molecular weight molecules, or polymers. The hydrolysable tannins can be further subdivided into gallotannins and ellagitannins depending on the occurrence and prevalence of either gallic acid or ellagic acid (gallic acid dimer) as a repeating building block ${ }^{8,15}$ Condensed tannins or proanthocyanidins are also high molecular weight polymers based on the monomeric building block flavan-3-ol. Interestingly, some condensed tannins such as the proanthocyanidins can occur as polymers with fifty or more repeating units and a mean molecular weight of $\sim 5,000 \mathrm{Da}$.

\section{Dietary intake of plant polyphenols}

Plant polyphenols are commonly consumed mostly from fruits, vegetables, legumes, wine and tea. Although widespread in the food supply, the quantification of dietary polyphenol content and estimated dietary intake has been complicated by several factors. Food composition databases remain incomplete, and often inadequate, providing only limited information on polyphenol content. Moreover, polyphenol content between foods is highly variable and even within a specific food item the polyphenol content can vary considerably and by several orders of magnitude. For example, the procyanidin content of four common varieties of apples has been reported to range from 12.3 to $252.4 \mathrm{mg} /$ serving, with the highest amounts found on average for Red Delicious and lowest for Golden Delicious varieties. ${ }^{16}$ Lastly, due to their polymeric composition and the myriad of combinations, poorly elucidated chemical structures, and lack of commercially available standards, analysis and estimation have been problematic. ${ }^{17,18}$

Besides plant variety, many other factors are important in determining polyphenol concentrations of specific food 
products in the food supply, including production method (organic/conventional), annual climatic variations, and post-harvest processing and storage. ${ }^{19}$ In a three-year comparison of the polyphenol contents of organically and conventionally produced apples, significantly higher polyphenol concentrations were found in the organically grown apples in the year 2005, but no significant differences were observed in 2004 and 2006. ${ }^{20}$ Annual environmental differences, particularly the annual climate, can also profoundly affect polyphenol production regardless of production method.

Differences in post-harvest processing and storage can greatly impact the content of phenolic acids and flavonoids in a variety of foods. ${ }^{19}$ For example, the flavanone content of market-ready orange juice varies with processing, with the highest amounts in traditional pasteurized juice and the lowest levels in frozen juice. ${ }^{21}$ Overall, the polyphenol content of juices is usually $200-500 \mathrm{mg} / \mathrm{L}$. The cooking method can also affect the polyphenol content of foods since polyphenols are present in plant vacuoles and apoplasts, cellular structures that require softening during the cooking process to maximize polyphenol release. ${ }^{22}$ However, vegetables such as onions, tomatoes and carrots can lose $75 \%$ to $100 \%$ of their initial polyphenol content during boiling whereas steaming and frying losses are considerably lower. ${ }^{22,23}$

Despite the numerous difficulties in quantifying the polyphenol content of foods, the US Department of Agriculture has released two food databases that include content of six subgroups of flavonoids, the most common polyphenols consumed in plant foods. These databases were used to estimate dietary flavonoid intake among Americans using the 1992-2002 National Health and Nutrition Examination Survey (NHANES) ${ }^{24}$ Intake was estimated at $189.7 \pm 11.2 \mathrm{mg}$, with tea, citrus fruit juices, wine, and citrus fruits being the major dietary sources. ${ }^{24}$ Fruits such as apple, grape, pear, cherry, and various berries have 200-300 mg polyphenols per $100 \mathrm{~g}$ fresh weight. ${ }^{25}$ Daily intake of polyphenols in coffee drinkers is $500-1,000 \mathrm{mg}$ since a cup of coffee contains around $100 \mathrm{mg}$ polyphenols. ${ }^{25}$ Chlorogenic acid, an ester of caffeic acid and quinic acid, is a major phenolic compound in coffee. In a study by Chun and colleagues, the estimated daily intake of polyphenols in the US was $449.8 \mathrm{mg}$ using per capita consumption data for 34 commonly consumed fresh fruits and vegetables. ${ }^{26}$ Following the recommendation to eat five servings of fruits and vegetables daily would result in a total polyphenol intake of $>500 \mathrm{mg}$ and a flavonoid intake of about $150-300 \mathrm{mg} /$ day. ${ }^{9}$ Depending on food choice, this could be much higher. For example, consumption of cocoa, tea or coffee as rich sources of hydroxycinnamic acids could easily increase overall intake by $500-1,000 \mathrm{mg} .{ }^{9}$ This would result in a $\sim 100$ times higher intake than that accomplished by the intake of a typical Western diet. In the Dutch diet, it is estimated that total intake of polyphenols is $863 \pm 415 \mathrm{mg} /$ day. ${ }^{27}$ Thus, it is not surprising that other estimates of daily intake are $\sim 1,000-1,100 \mathrm{mg} /$ day depending on the season. Although dietary consumption of polyphenols is high, the risk of toxicity from the food supply is relatively low, largely due to poor absorption. However, concentrations of dietary bioactive agents can be significantly increased, and presumably absorption as well, through food-based polyphenol enrichment, alterations in bioavailability, and supplementation with purified agents or mixtures rendering polyphenol consumption potentially problematic.

\section{Dietary supplements}

A significant percentage of the population in Western and developing countries are not consuming sufficient quantities of dietary polyphenols as a result of inadequate fruit and vegetable intakes, although a large body of evidence mainly derived from preclinical studies has concluded that dietary polyphenols, given in large quantities, can have desirable outcomes. ${ }^{28}$ A dietary supplement could provide some of the same beneficial effects of dietary polyphenols in the face of minimal consumption of fruits and vegetables. As such, many in the population embrace the notion of supplementation with polyphenols, such as flavonoids, in the hopes of achieving more beneficial effects.

Numerous surveys such as the NHANES show that dietary supplements are used by a large proportion $(68 \%)$ of the general public primarily, through self-reporting, to improve overall health. Most use traditional and well-known vitamins and minerals such as the water and fat-soluble vitamins and calcium and iron. Non-nutritive dietary supplements are also frequently used and include polyphenol-rich green tea, fish oil, echinacea, glucosamine, isoflavone-rich soy, flax seed, and chondroitin. ${ }^{29}$ In a survey of health professionals by the Council for Responsible Nutrition, it was shown that $72 \%$ of physicians and $89 \%$ nurses consumed dietary supplements regularly. Use by the general population was estimated to be $59 \%$. Medical personnel stated their rationale for use and subsequent recommendation to patients $(>80 \%$ reporting recommending) was based on information reported and disseminated in professional journals and clinical studies.

Overall, the beneficial outcomes reported for vitamins and minerals, as well as polyphenols, has contributed to the significant popularity of dietary supplements. In fact, this has led to the development and marketing of new polyphenol 
dietary supplements and polyphenol-rich food products. The result is increased intake of particular polyphenols alone or in combination, beyond levels of exposure commonly associated with a typical diet. Based on recommendations by the dietary supplement industry, it is advised to consume $50 \mathrm{mg} /$ day isoflavones or $100-300 \mathrm{mg} /$ day grape seed extracts rich in proanthocyanidins. ${ }^{30}$ According to Mennen and colleagues, examples of commonly recommended doses of polyphenols found on the internet are $300 \mathrm{mg}$ quercetin, $1 \mathrm{~g}$ citrus flavonoids or $20 \mathrm{mg}$ resveratrol with suggested use of 1-6 capsules per day. ${ }^{30}$ This would result in $~ 100$ times higher intake that the common intake of a Western diet.

\section{Bioavailability of polyphenol compounds}

Dietary intake of plant polyphenols can vary considerably and be quite high, ie, g/day, but ultimately the nutritional impact and subsequent systemic effects depend on the fate of polyphenols in the digestive tract. Physicochemical characteristics of polyphenols appear to dictate absorption and metabolism and are determined primarily by chemical structure. For example, the parent structure (gallic acid, ellagic acid, flavan-3-ol), degree of chemical modification, ie, glycosylation, conjugation, etc, molecular size, degree of polymerization, and solubility are critical factors. ${ }^{3}$ Relatively small molecular weight phenolic acids, ie, gallic acid and isoflavones are easily absorbed through the gut followed by catechins, flavanones, and quercetin glucosides. Larger polyphenols such as proanthocyanidins are very poorly absorbed as well as galloylated tea catechins and the anthocyanins.

The main difficulty with incorporating polyphenols into existing products is to present them in a form that is bioavailable. Bioavailability has been estimated in rodent studies to be $\leq 10 \%$ of ingested dose with a range of $2 \%-20 \%{ }^{28}$ Data in humans are limited by the need for large population sizes, however, Manach and colleagues has reviewed bioavailability of 18 major polyphenols in 97 human bioavailability studies and reported that plasma concentrations of total polyphenol metabolites ranged from 0-4 $\mu \mathrm{mol} / \mathrm{L}$ with an intake of $50 \mathrm{mg}$ aglycone equivalents and the urinary excretion ranged from $0.3 \%-43 \%$ of the ingested dose. ${ }^{25}$ It was further noted that bioavailability differed greatly from one polyphenol to another and the most abundant dietary polyphenol was not necessarily the one leading to the highest levels of active metabolites in plasma. Kroon and colleagues have reported maximum plasma concentrations of $0.1-10 \mu \mathrm{mol} / \mathrm{L} .{ }^{31}$ It has also been noted that plasma levels of conjugated flavonoids rarely, if at all, exceed $1 \mu \mathrm{mol} / \mathrm{L} .{ }^{32}$
Numerous human pharmacokinetic studies have determined plasma and urine concentrations after specific doses of polyphenols. For example, when quercetin, a flavanol, was provided from onions and ketchup at $90 \mathrm{mg}$ /day for two weeks, plasma levels increased to $0.289 \mu \mathrm{mol} / \mathrm{L} .{ }^{33}$ Urinary excretion was $0.262 \mathrm{mg}$ over 24 hours. When naringenin, a flavanone, derived from either orange or grapefruit juices, was given as a single 23 or $199 \mathrm{mg}$ dose, respectively, plasma levels increased to 0.64 and $5.98 \mu \mathrm{mol} / \mathrm{L}$, respectively. ${ }^{34}$ Epigallocatechin from green tea extracts was given as a single $17.5 \mathrm{mg}$ dose and plasma concentrations increased to $0.025 \mu \mathrm{mol} / \mathrm{L} .{ }^{35}$ Interestingly, recent studies in humans have demonstrated that fasting further increases bioavailability of epigallocatechin gallate (EGCG). ${ }^{36}$ When epicatechin from chocolate was provided as a single $104 \mathrm{mg}$ dose, plasma levels rose to $0.676 \mu \mathrm{mol} / \mathrm{L}^{37}$ Anthocyanins given as a single dose of $20 \mathrm{mg} / \mathrm{kg}$ body weight from blackcurrant juice increased plasma levels to $0.155 \mu \mathrm{mo} / \mathrm{L} .{ }^{38} \mathrm{~A}$ single $2.7 \mathrm{mg}$ dose of caffeic acid from red wine increased plasma levels, one hour post-ingestion, to $0.027 \mu \mathrm{mol} / \mathrm{L} .{ }^{39}$ Clearly, absorption and ultimately bioavailability of polyphenols vary greatly and are complex.

The critical importance of bioavailability in conferring additional benefit or toxicity depends on increased absorption of polyphenols. This has fostered categorization of polyphenols as readily absorbed or not, alternately defined as extractable or non-extractable. ${ }^{3}$ Extractable polyphenols are low to intermediate molecular weight phenolics, which can be extracted with different, commonly used solvents such as water or methanol. Nonextractable polyphenols are high molecular weight compounds that can bind dietary fiber or protein and remain insoluble in the aforementioned solvents. As such, they tend to be relatively resistant to intestinal digestion and/ or absorption and are readily excreted in feces.

The physicochemical characteristics of polyphenols likely contribute to the disparity in results between the epidemiological studies and in vitro data, which may reflect, in part, relatively poor bioavailability, rapid metabolism, and excretion of polyhenols. ${ }^{13}$ Efforts are being attempted to increase bioavailability but several considerations, and potential impediments, exist including solubility, permeability, metabolism, excretion, target tissue uptake, and disposition. ${ }^{28}$ Initial strategies for improving bioavailability of dietary polyphenol supplements include changes to polyphenol structure, which may affect solubility and dissolution, and the use of pharmaceutical excipients. ${ }^{28}$ An additional consideration is that degradation and absorption of polyphenols within the gastrointestinal tract 
depend on the intestinal microflora and gut enzymes, which may significantly change bioavailability.

\section{Metabolism of polyphenols}

Dietary polyphenols are initially metabolized, as many xenobiotics, within the gastrointestinal tract. Glycosides are hydrolyzed to their corresponding aglycones prior to absorption and aglycones and low molecular weight polyphenols are absorbed directly. ${ }^{3,25}$ Polyphenols that are not absorbed are transported to the colon where they can be readily hydrolyzed by colonic microflora to simpler chemical compounds and eliminated in the feces or further modified. Bacterial fermentation of the glycones effectively releases the polyphenol from fiber permitting subsequent metabolism similar to other aglycones. ${ }^{3}$ The high molecular weight polyphenols such as the ellagitannins are not absorbed intact into the bloodstream but are hydrolyzed to ellagic acid and urolithins and then absorbed, conjugated in the liver, and either excreted in the urine or stored in tissues, ie, prostate, etc. ${ }^{40,41}$ Post-absorption aglycones are methylated by catechol-O-methyltransferase (COMT) and/or conjugated with glucuronic acid or sulfate in the liver via the action of UDP glucurosyl transferase (UDPGT) or phenol sulfotransferases, respectively. ${ }^{25,42}$ Conjugated and 3'-O-methylated compounds have also been detected in plasma and are readily excreted in urine or bile. Polyphenolic compounds secreted in bile may also recycle, after deconjugation, through enterohepatic circulation and be reabsorbed.

Pharmacokinetic studies of the absorption and elimination of polyphenols indicate that maximum blood levels generally occur at $\sim 2$ hours post-ingestion and the elimination halflife is $\sim 4.8-6.9$ hours. $^{3}$ Seeram et al have found in a small human study that ellagitannin metabolites from ingested pomegranate juice persist up to 48 hours in the plasma and urine. ${ }^{41}$ Clearly, these values may vary considerably and likely due to the chemical nature, size, and complexity of the polyphenol.

\section{Protection by dietary polyphenols}

Using diverse in vitro cellular models, polyphenols exhibit powerful antioxidant activities and have been consistently protective through scavenging numerous diverse reactive oxygen species (ROS) including hydroxyl radical, peroxyl radical, hypochlorous acid, superoxide anion, and peroxynitrite. ${ }^{32}$ Examples of cell models previously used include human prostate cells, colon cancer cells, hepatocytes, leukemia cells, breast cancer cells, and oral epithelial cells. ${ }^{43}$ Mechanisms of action have included antioxidant effects, cardio- and hepatoprotective effects, anticarcinogenic effects, antimicrobial and antiviral effects, and anti-inflammatory effects. . $^{3,7,8}$

Although antioxidant activity traditionally has been attributed only to soluble phenolic compounds, or extractable polyphenols, a recent report suggests that nonextractable polyphenols (polymeric proanthocyanidins and high molecular weight hydrolysable tannins) are 15-30 times more effective at quenching peroxyl radicals than are simple phenols. ${ }^{3}$ Moreover, those that are not absorbed could exert effects in the gastrointestinal tract protecting macromolecules, ie, lipids, proteins, and carbohydrates.

Largely, however, the antioxidant efficiency of polyphenols depends on the extent of absorption and metabolism of these compounds as well as the activity of conjugates circulating in plasma. As discussed previously, bioavailability from dietary intake of polyphenols is $2 \%-20 \%$ so minimal amounts of dietary polyphenols are absorbed, with subsequently low levels found in plasma. However, low concentrations seem adequate to exert potent protective antioxidant activity in vivo as suggested by epidemiological data.

Although the most frequently reported benefit of polyphenols involves their antioxidant activity, there are numerous other biological processes that are affected by polyphenols. For example such processes include; altered gene expression, increased apoptosis, decreased platelet aggregation, increased blood vessel dilation, modulated intercellular signaling, P-glycoprotein activation, and the modulation of enzyme activities associated with carcinogen activation and detoxification. ${ }^{44}$ Additionally, polyphenols can chelate transition metals, ie, iron, decreasing their ability to promote ROS formation through Fenton chemistry. ${ }^{45,46}$

\section{Adverse pro-oxidant effects of high-dose polyphenols}

A considerable body of literature supports a role for oxidative stress in the pathogenesis of age-related human disease and a role for dietary polyphenols in prevention. ${ }^{25}$ Diet-derived compounds are generally regarded as safe, based on their long history of use. It is becoming apparent, however, that commonly used dietary compounds can exert deleterious effects at pharmacological concentrations. This is further exacerbated by the increased vulnerability of certain populations, the co-existence of additional diseases, and/or interaction with other pharmacologic agents. ${ }^{47}$

Dietary polyphenols are purportedly potent antioxidants as demonstrated, in large part, through in vitro studies. Demonstration in vivo has been problematic although an increasing body of evidence suggests detrimental pro-oxidant effects. 
Under certain conditions (high concentrations of phenolic antioxidants, high $\mathrm{pH}$, presence of iron) phenolic antioxidants can initiate an auto-oxidation process and behave like pro-oxidants. ${ }^{48}$ Hot beverages contain high $\mathrm{H}_{2} \mathrm{O}_{2}$ concentrations because polyphenols oxidize rapidly at high temperatures. Probably the most compelling supportive evidence involves studies of EGCG commonly found in tea. $\mathrm{H}_{2} \mathrm{O}_{2}$ has been measured up to $10 \mu \mathrm{mol} / \mathrm{L}$ in saliva of those chewing green tea leaves. ${ }^{49}$ EGCG undergoes, in vitro, oxidative polymerization and generation of toxic $\mathrm{H}_{2} \mathrm{O}_{2}$ at concentrations up to $334 \mu \mathrm{mol} / \mathrm{L}$ when adding $1 \mathrm{mmol} / \mathrm{L}$ EGCG to culture medium. ${ }^{48,50,51}$ Addition of catalase, an enzyme that specifically quenches $\mathrm{H}_{2} \mathrm{O}_{2}$, inhibits the death of human lung cancer cells incubated with EGCG. In hepatocytes given high-dose EGCG (200 $\mu \mathrm{mol} / \mathrm{L})$, cell viability was significantly reduced and associated with increased ROS production and antioxidant, viz, glutathione, depletion. ${ }^{42}$ In human lung and esophageal cancer cells, EGCG ( $20 \mu \mathrm{mol} / \mathrm{L}$ for 24 hours $)$, presumably via ROS production, interrupted phosphorylation of epidermal growth factor receptor and the second messenger signaling cascade. This was attenuated by addition of antioxidant enzymes including superoxide dismutase (SOD) and catalase. ${ }^{52}$

Interruption of cell signaling cascades can be detrimental to cells and contribute to chronic disease. Indeed, phytoestrogenic modulation of hormonal signaling by soy-based isoflavones, such as genistein, daidzein, and glycitein, have generated considerable debate on the demonstration of both adverse and detrimental effects in breast cancer. ${ }^{53-55}$ Epidemiological data support an inverse relationship between increased isoflavone consumption and a decreased risk of breast cancer. However, data also suggest that dietary isoflavones can promote breast cancer. ${ }^{53}$ For example, resveratrol, a polyphenol stilbene in red wine and available as a dietary supplement, can bind the estrogen receptor and foster breast cancer cell growth at concentrations of $0.1-1000 \mu \mathrm{mol} / \mathrm{L} .{ }^{56}$ However, this effect is biphasic depending on the estrogen concentrations. Resveratrol is protective in microenvironments with high hormone (estrogens and androgens) concentrations, but stimulatory in low hormone environments. The isoflavone genistein also inhibits the growth of most cancer cells but in estrogen-responsive $\left(\mathrm{ER}^{+}\right)$cells the response is biphasic where genistein inhibits human breast cancer cell growth at higher concentrations yet stimulates proliferation of $\mathrm{ER}^{+}$ breast cancer cells at lower concentrations consistent with physiologically relevant levels in humans. In an in vivo rodent model, ovariectomized athymic mice fed genistein and genistin both displayed enhanced growth of $\mathrm{ER}^{+}$mammary tumors. ${ }^{57,58}$
In human studies, premenopausal women consuming isoflavone-rich isolated soy protein displayed 2-6-fold increases in the volume of nipple aspirate supporting an estrogen-induced effect. ${ }^{59}$ Moreover, histological examination revealed that nearly one-third of the subjects (7 of 24) consuming soy displayed epithelial hyperplasia, which is strongly associated with breast cancer. In a different study, premenopausal women consuming soy textured protein for two weeks revealed increased estrogen-regulated pS2 levels in breast biopsies indicative of a phytoestrogen response. ${ }^{60}$ Clearly, more research is needed to better understand the effects, whether positive or negative, of isoflavones and other polyphenols particularly in the context of dose, timing, duration, and susceptibility of consumer to disease, ie, breast cancer. This is particularly relevant given the widespread availability of dietary supplements of isoflavones and soy-based formulations.

Phenolic acids comprise $\sim 30 \%$ of dietary polyphenols. High-dose phenolic acids promote carcinogenicity in some studies through $\mathrm{H}_{2} \mathrm{O}_{2}$-induced DNA damage and resultant genotoxicity. ${ }^{61}$ Moreover, studies show administration of high doses (up to $50 \mu \mathrm{mol} / \mathrm{L}$ ) of purified flavonoids for six hours could cause chromosome translocation and clastogenicity in human cell line studies. ${ }^{47,62}$ Thus, it is proposed from these studies that phenolic acids can enhance tumor promotion, or increase proliferative signaling, via ROS production, as previously discussed, through interruption of second messenger signaling.

In many studies, the effect of polyphenol dose leading to toxicity becomes apparent. Ferulic acid, a phenolic acid, is an antioxidant which neutralizes free radicals (superoxide, nitric oxide and hydroxyl radical) and is found in the leaves and seeds of many plants and cereals such as brown rice, whole wheat and oats, coffee, apple, artichoke, peanut, orange and pineapple. ${ }^{63}$ Research results have indicated that ferulic acid when fed to rats at $500 \mathrm{mg} / \mathrm{kg}$ diet is carcinogenic to liver but anticarcinogenic to tongue, skin and colon. ${ }^{64}$ Dietary caffeic acid, a precursor of ferulic acid, has been shown to be carcinogenic at $2 \%$ (20 g/ $\mathrm{kg}$ diet ), a tumor promoter at $0.5 \%-1 \%$ $(5-10 \mathrm{~g} / \mathrm{kg} \mathrm{diet})$, and anticarcinogenic at $0.05 \%-0.5 \%$ $\left(0.5-5 \mathrm{~g} / \mathrm{kg}\right.$ diet) ${ }^{64-66}$ Thus, it appears that the dose, which is closely associated with bioavailability, contributes to both beneficial and adverse effects with higher doses contributing to the latter.

\section{Animal studies}

Numerous in vivo animal studies have demonstrated adverse effects of dietary polyphenols. Administration 
of a polyphenol-rich green tea preparation (90\% EGCG) for 13 weeks to dogs produced dose-dependent toxicity and death. ${ }^{67}$ Furthermore, dietary levels of $500 \mathrm{mg} / \mathrm{kg}$ caused proximal tubule necrosis, elevated serum bilirubin, and increased aspartate aminotransferase (AST), in all animals tested, indicating severe kidney and liver damage. ${ }^{67}$ In rodents, a polyphenol supplement given orally $(2 \mathrm{~g} / \mathrm{kg})$ by gastric gavage caused lethality in $80 \%$ of the animals treated with histological evidence of hemorrhagic lesions in both the stomach and intestine. Intraperitoneal administration of EGCG to rodents caused dose-dependent lethality and increased liver damage at doses as low as $150 \mathrm{mg} / \mathrm{kg}(10.5 \mathrm{~g}$ for a $70 \mathrm{~kg}$ human). ${ }^{42}$ Collectively, high-dose EGCG induced multi-organ toxicity associated with increased plasma levels beyond that normally encountered through diet. Bioavailability of polyphenols is relatively low in rodents, thus adverse effects were limited to the gastrointestinal tract. In contrast, bioavailability is much higher in canines, and, as a result, pathology was noted in the gastrointestinal tract, the liver and kidneys highlighting the importance of absorption and resultant plasma concentration. ${ }^{47}$

EGCG can undergo redox cycling and produce quinones with subsequent increases in oxidative stress. For example, intraperitoneal injection of EGCG led to formation of cysteine conjugates formed only at high doses of $200-400 \mathrm{mg} / \mathrm{kg}$, supporting the likely role of oxidative stress, free radicalassociated quinone formation. Second, EGCG treatment of lung cancer-bearing nude mice for 40 days with $40 \mathrm{mg} / \mathrm{kg}$ EGCG, given intraperitoneally increased markers of DNA damage and oxidative stress. ${ }^{47}$

Chemical structures and molecular size are important determinants of effect. For example, no acute toxicity was noted in rodents after administration of high molecular weight polyphenols including oral grape seed proanthocyanidin extract $(0.5-2.0 \mathrm{~g} / \mathrm{kg}$ body weight) or the ellagitannin punicalagin $(60 \mathrm{~g} / \mathrm{kg}){ }^{68,69}$ In the first study, rats were fed ad libitum for 37 days either a commercial diet alone or containing $20 \%$ (w/w; $200 \mathrm{~g} / \mathrm{kg}$ diet) pomegranate husk extract to reach an average $6 \%(\mathrm{w} / \mathrm{w})$ punicalagin concentration, or $60 \mathrm{~g} / \mathrm{kg}$ diet, in the final diet. ${ }^{68}$ This amount translated to consumption by rats of $0.9 \mathrm{~g}$ punicalagin per day. Plasma levels of punicalagin peaked at $29 \mu \mathrm{g} / \mathrm{mL}$ plasma $(27 \mu \mathrm{mol} / \mathrm{L})$ and a higher dietary intake did not further increase plasma levels. The authors report that this level was equivalent in humans to consumption of $\sim 194 \mathrm{~L}$ of pomegranate juice per day suggesting a wide safety margin. ${ }^{69}$ In the second study, grape seed proanthocyanidins were administered orally by gastric intubation to male and female albino rats at $10 \mathrm{~mL} / \mathrm{kg}$ (average body weight $0.25 \mathrm{~kg}$ ). One group was given $5 \mathrm{~g} / \mathrm{kg}$ body weight. grape seed proanthocyanidin extract (GSPE) was also fed to groups of rats at $100 \mathrm{mg} / \mathrm{kg}$ body weight for 12 months with testing of one group every three months. The lethal dose for $50 \%$ of the group $\left(\mathrm{LD}_{50}\right)$ was greater than $5 \mathrm{~g} / \mathrm{kg}$ when administered orally by gavage and $2 \mathrm{~g} / \mathrm{kg}$ was the highest dose with no observed adverse effects. Chronic administration for 12 months did not cause pathology in any of the seven vital organs tested. Plasma levels were not provided but the equivalent for dietary levels associated with the chronic study for humans would be $7 \mathrm{~g}(100 \mathrm{mg} /$ day; $70 \mathrm{~kg}$ human).

Quercetin is a plant-derived flavonoid, viz, a flavanol, used as a dietary supplement purportedly possessing antiinflammatory and antioxidant properties. ${ }^{12,25}$ Low molecular weight, presumably better absorbed quercetin, was fed for two years to male and female $\mathrm{F} 344 / \mathrm{N}$ rats at concentrations of $2 \%(20 \mathrm{~g} / \mathrm{kg}$ diet $)$ and $4 \%(40 \mathrm{~g} / \mathrm{kg}$ diet $)$. The estimated dose delivered was $\sim 40-1,900 \mathrm{mg} / \mathrm{kg}$ body weight/day. There were no quercetin-associated adverse effects on survival and no clinical signs of toxicity although histological examination revealed chronic nephropathy at these doses. ${ }^{70}$ Quercetin significantly inhibited metabolism of substrates prone to redox cycling and increased pro-oxidant formation leading to high, toxic levels in organs and tissues. ${ }^{71}$ In this study, four-week-old male hamsters were fed diet with $3 \%$ $(30 \mathrm{~g} / \mathrm{kg}$ diet) quercetin for two weeks and 6.5 months. Plasma levels were similar in both studies and reached $\sim 0.22 \mu \mathrm{mol} / \mathrm{L}$ $(74.4 \mathrm{ng} / \mathrm{mL}$ ). Average daily intake of quercetin is $\sim 25 \mathrm{mg}$. Studies with caffeic acid ( $2 \%$ of diet) support these findings since administration induces forestomach and kidney tumors in rodents at normal dietary levels presumably by interruption of metabolism of estrogenic substrates. Furthermore, it has been reported in a review by Mennen and colleagues that tissues rich in oxidative enzymes may be especially vulnerable to pro-oxidant toxicity by quercetin. ${ }^{30}$

\section{Human studies}

Antioxidants, including polyphenols, have been linked to numerous health benefits but may be detrimental when taken in larger doses found in dietary supplements and fortified foods. Some human intervention trials have shown not only failure to protect by polyphenols but accelerated development of cancers or CVD in susceptible subjects. ${ }^{32}$ There have been a number of recent case reports of hepatotoxicity related to the consumption of high doses of polyphenolenriched, tea-based dietary supplement $(10-29 \mathrm{mg} / \mathrm{kg} / \mathrm{d}){ }^{72}$ For example, a 37-year-old Hispanic female consuming a 
green tea supplement for four months displayed elevated blood chemistry levels of AST (1,783 U/L; normal 17-39), alanine transferase (ALT; 1,788 U/L; normal 8-39), total bilirubin $(200 \mu \mathrm{mol} / \mathrm{L} ; 7-24)$, direct bilirubin $(169 \mu \mathrm{mol} / \mathrm{L}$; $0-3$ ), and serum alkaline phosphatase (ALP; $238 \mu \mathrm{mol} / \mathrm{L}$; 39-113) indicating serious liver dysfunction. A liver biopsy revealed marked interfacial necrosis and inflammation including mild lobular inflammation. After discontinuing the polyphenol-rich dietary supplement, the patient's values returned to normal. However, after a subsequent re-challenge and exclusion of other causes, signs and symptoms returned strongly suggesting that the high-dose dietary supplement (383.3 mg per three capsules) was causing severe, symptomatic drug-induced liver injury. The conclusion was that concentrated preparations of polyphenol-rich green tea were dangerous and should be avoided. ${ }^{72}$

Several additional human case studies have shown hepatotoxicity related to the consumption of high-dose, teabased dietary supplements (10-29 mg/kg/day per os). In nearly eight of nine cases patients presented with elevated ALT and bilirubin levels. In two of nine cases, periportal and portal inflammation was observed in the liver. All pathology resolved after the dietary supplement was discontinued although reinjury occurred with rechallenge. For the case studies, it is noteworthy that susceptible individuals may have genetic variations, or polymorphisms, in critical biotransformation pathways for tea polyphenols, ie, catecholO-methyltransferase. ${ }^{47}$

Inclusion or exclusion of polyphenol-enriched, or fortified, foods can also lead to potentially adverse effects. Consumption of flavonoid-free diets by human volunteers has recently been reported to decrease markers of oxidative stress commonly associated with chronic disease. ${ }^{73}$ Consumption of antioxidant-rich foods increases biomarkers of oxidative damage and biomarkers of protein damage such as 2-aminoadipic and gamma glutamyl semialdehydes. ${ }^{73}$ Small and transient increases in oxidative DNA damage have been noted in human volunteers fed mixtures of other antioxidants such as ascorbic acid, beta carotene, and alpha tocopherol. ${ }^{74}$

Polyphenols can exhibit many adverse effects to diverse biological systems. For example, several flavonoids inhibit the enzyme thyroid peroxidase and interfere with thyroid hormone biosynthesis and ultimately thyroid function. Reports also suggest that elevated consumption of soy isoflavones can reduce fertility and retard sexual maturation. Polyphenols can also exhibit antinutritional effects through complexation of minerals such as iron. While this can be protective, it can also lead to iron deficiency., ${ }^{3,75}$ Larger molecular weight polyphenols, ie, tannins, can interact with proteins and inhibit several enzymes that are needed for growth. High polyphenol intake could also increase the risk of CVD through alterations in homocysteine processing, a well-accepted biomarker for CVD. ${ }^{76,77}$ Lastly, polyphenols, specifically naringenin in grapefruit, can inhibit drug-metabolizing enzymes such as CYP3A4, among others, involved in xenobiotic metabolism and, thus, interact with pharmacological agents increasing the risk of overdose and harm. ${ }^{78}$

The effect of grapefruit is particularly relevant since a large portion of the population are prescribed $\beta$-blockers or cholesterol-lowering statins to manage dyslipidemia associated with cardiovascular disease. ${ }^{79,80}$ Grapefruit juice is specifically contraindicated to avoid cardiovascular complications since naringenin, naringin, and 6,7-dihdroxybergamottin, as grapefruit-derived bioactive agents, can inhibit the CYP3A4 enzyme and alter the pharmacokinetics of medications leading to marked increases in their plasma concentrations and ultimate toxicity. ${ }^{81-83}$ While data support this contention, grapefruit juice has traditionally been shown to contain antioxidant, antinitrosaminic, antiseptic, cardiotonic, hypocholesteromic, and sedative activities among several other functions all of which are positive, beneficial effects. ${ }^{78}$ As such, it may be prudent that those on medications requiring CYP3A4 metabolism refrain from consumption. It must be pointed out that many diverse polyphenols can affect the activities of many CYP450 enzymes other than 3A4 by induction and inhibition. For example, quercetin, flavone, and diosmin, as examples, can induce CYP1A1, whereas kaempferol, ellagic acid, and resveratrol can inhibit CYP1A1. ${ }^{84}$

\section{Conclusions}

Pro-oxidant-induced oxidative stress contributes to the pathogenesis of numerous chronic diseases and, as such, dietary antioxidants can and should quench and/or retard such processes. However, considerable evidence supports that high-dose polyphenols can potentially cause adverse effects through pro-oxidative effects. As such, the medical literature is replete with reports of the beneficial properties of dietary polyphenols, but also the possible adverse effects of polyphenol-rich plants and their components as fortified, enhanced, or purified foods and/or dietary supplements. ${ }^{85}$

Risk is increased by the use of pharmacological doses of polyphenols in prevention, treatment and dietary supplement situations. Bioavailability appears to be a regulatory mechanism of sorts in keeping the overall absorption of polyphenols low, ie, 2\%-20\%. Given that overwhelming 
evidence supports intake of polyphenols in disease prevention and attenuation, efforts are being made to not only increase dietary intake but to increase bioavailability by increasing absorption of polyphenols singly or in combination.

Indeed, dietary supplements that contain high (pharmacologic) doses of polyphenols can, in fact, be developed. As a result, intake of polyphenols can easily reach very high levels and presumably plasma and/or tissue levels will also reach higher levels not normally encountered in the typical diet. As such, testing is prudent to ensure safety and the low risk of adverse effects from this level of intake. Given the complexity and breadth of polyphenols as a chemical family, it may be wise to start with assessing the nature of the polyphenol-rich dietary product (fortified or enhanced food, food extract, or pure compound, ie, dietary supplement) and its intended use. It has been suggested that the addition of specific polyphenols, or combinations, to different foods should be strictly controlled to limit consumption to a dosage window where health benefits, and lack of adverse effects, has been shown. ${ }^{25}$

\section{Disclosures}

The authors report no conflicts of interest in this work.

\section{References}

1. Lock K, Pomerleau J, Causer L, Altman DR, McKee M. The global burden of disease attributable to low consumption of fruit and vegetables: implications for the global strategy on diet. Bull World Health Organ. 2005;83:100-108.

2. Fraga CG. Plant polyphenols: how to translate their in vitro antioxidant actions to in vivo conditions. IUBMB Life. 2007;59:308-315.

3. Bravo L. Polyphenols: Chemistry, dietary sources, metabolism, and nutritional significance. Nutr Rev. 1998;56:317-333.

4. Liu RH. Potential synergy of phytochemicals in cancer prevention: mechanism of action. J Nutr. 2004;134:3479S-3485S.

5. Urquiaga I, Leighton F. Plant polyphenol antioxidants and oxidative stress. Biol Res. 2000;33:55-64.

6. Weisburger JH. Chemopreventive effects of cocoa polyphenols on chronic diseases. Exp Biol Med. 2001;226:891-897.

7. Nijveldt RJ, van Nood E, van Hoorn DEC, Boelens PG, van Norren K, van Leeuwen PAM. Flavonoids: A review of probable mechanisms of action and potential applications. Am J Clin Nutr. 2001;74:418-425.

8. Serrano J, Puupponen-Pimia R, Dauer A, Aura AM, Saura-Calixto F. Tannins: Current knowledge of food sources, intake bioavailability and biological effects. Mol Nutr Food Res. 2009;53:1-20.

9. Williamson G, Holst B. Dietary reference intake (DRI) value for dietary polyphenols: Are we heading in the right direction? Br J Nutr. 2008;99(3):S55-S58.

10. Tapiero H, Tew KD, Ba GN, Mathe G. Polyphenols: do they play a role in the prevention of human pathologies? Biomed Pharmacother. 2002;56:200-207.

11. Hollman PCH, Arts ICW. Flavonols, flavones and flavanols-Nature, occurrence and dietary burden. J Sci Food Agric. 2000;80:1081-1093.

12. Ross JA, Kasum CM. Dietary flavonoids: Bioavailability, metabolic effects, and safety. Ann Rev Nutr. 2002;22:19-34.

13. Duthie GG, Gardner PT, Kyle JAM. Plant polyphenols: are they the new magic bullet? Proc Nutr Soc. 2003;62:599-603.
14. Williamson G. Antioxidant advances. Food Technol Intl. 2008:30-31.

15. Clifford MN, Scalbert A. Ellagitannins-Nature, occurrence and dietary burden. J Sci Food Agric. 2000;80:1118-1125.

16. Hammerstone JF, Lazarus SA, Schmitz HH. Procyanidin content and variation in some commonly consumed foods. J Nutr. 2000;130(8S): 2086S-2092S.

17. Seeram NP. Bioactive polyphenols from foods and dietary supplements: Challenges and opportunities. In: Series ACSS, editor. Herbs. Vol. 925. Washington, DC: American Chemical Society; 2006:25-38.

18. Martin KR, Krueger CG, Rodriguez G, Dreher M, Reed JD. Development of a novel pomegranate standard and new method for the quantitative measurement of pomegranate polyphenols. J Sci Food Agric. 2008;89:157-162.

19. Amarowicz R, Carle R, Dongowski G. Influence of postharvest processing and storage on the content of phenolic acids and flavonoids in foods. Mol Nutr Food. 2009;53 (suppl 2):S151-S183.

20. Stracke BA, Rufer CE, Weibel FP, Bub A, Watzl B. Three-year comparison of the polyphenol contents and antioxidant capacities in organically and conventionally produced apples (malus domestica bork. cultivar golden delicious). J Agric Food Chem. 2009;57:4598-4605.

21. Sanchez-Moreno C, Plaza L, de Ancos B, Cano M. Quantitative bioactive compounds assessment and their relative contribution to the antioxidant capacity of commercial orange juices. J Sci Food Agric. 2003;83:430-439.

22. Miglio C, Chiavaro E, Visconti A, Fogliano V, Pellegrini N. Effects of different cooking methods on nutritional and physicochemcial characteristics of selected vegetables. J Agric Food Chem. 2008;56:139-147.

23. Crozier A, Lean MEJ, McDonald MS, Black C. Quantitative analysis of the flavonoid content of commercial tomatoes, onions, lettuce, and celery. J Agric Food Chem. 1997;45:590-595.

24. Chun OC, Chung SJ, Song WO. Estimated dietary flavonoid intake and major food sources of US adults. J Nutr. 2007;137:1244-1252.

25. Scalbert A, Manach C, Morand C, Remesy C, Jimenez L. Dietary polyphenols and the prevention of diseases. Criti Rev Food Sci Nutr. 2005;45:287-306.

26. Chun OK, Kim DO, Smith NL, Schroeder D, Han JT, Lee CY. Daily consumption of phenolics and total antioxidant capacity from fruits and vegetables in the American diet. J Sci Food Agric. 2005;85:1715-1724.

27. Orvaskainen ML, Torronen R, Koponen JM, et al. Dietary intake and major food sources of polyphenols in Finnish adults. $J$ Nutr. 2008; 138:562-566.

28. Hu M. Commentary: Bioavailability of flavonoids and polyphenols: Call to arms. Mol Pharm. 2007;4:803-806.

29. Dickinson A, Boyon N, Shao A. Physicians and nurses use and recommend dietary supplements: report of a survey. Nutr J. 2009; $8: 1-18$.

30. Mennen LI, Walker R, Bennetau-Pelissero C, Scalbert A. Risks and safety of poyphenol consumption. Am J Clin Nutr. 2005;81:326S-329S.

31. Kroon PS, Clifford MN, Crozier A, et al. How should we assess the effects of exposure to dietary polyphenols in vitro? Am J Clin Nutr. 2004;80:15-21.

32. Halliwell B. Dietary polyphenols: Good, bad, or indifferent for your health? Cardiovasc Res. 2007;73:341-347.

33. Young JF, Nielsen SE, Haraldsdottir J, et al. Effect of fruit juice intake on urinary quercetin excretion and biomarkers of oxidative stress. $\mathrm{Am}$ J Clin Nutr. 1999;69:87-94.

34. Vitaglione P, Sforza S, Galaverna G, et al. Bioavailability of transresveratrol from red wine in humans. Mol Nutr Food Res. 2005;49: 495-504.

35. Erlund I, Silaste ML, Alfthan G, Rantala L, Kesaniemi YA, Aro A. Plasma concentrations of the flavonoids hesperetin, naringenin, and quercetin in human subjects following their habitual diets, and diets high or low in fruits and vegetables. Eur J Clin Nutr. 2002;56:891-898.

36. Chow HH, Hakim IA, Vining DR, et al. Effects of dosing condition on the oral bioavailability of green tea catechins after single-dose administration of polyphenon $\mathrm{E}$ in healthy individuals. Clin Cancer Rres. 2005;11:4627-4633. 
37. Unno T, Kondo K, Itakura H, Takeo T. Analysis of (-)-epigallocatechin gallate in human serum obtained after ingesting green tea. Biosci Biotechnol Biochem. 1996;60:2066-2068.

38. Spencer JPE, Kuhnle GGC, Williams RJ, Rice-Evans C. Intracellular metabolism and bioactivity of quercetin and its in vivo metabolites. Biochem J. 2003;372:173-181.

39. Ritchie MR, Morton MS, Deighton N, Blake A, Cummings JH. Plasma and urinary phytoestrogens as biomarkers of intake: Validation by duplicate diet analysis. Br J Nutr. 2004;91:447-457.

40. Heber D. Multitargeted therapy of cancer by ellagitannins. Cancer Lett. 2008;269(2):262-268.

41. Seeram NP, Henning SM, Zhang Y, Suchard M, Li Z, Heber D. Pomegranate juice ellagitannin metabolites are present in human plasma and some persist in urine for up to 48 hours. J Nutr. 2006;136: 2481-2486.

42. Galati G, Lin A, Sultan AM, O'Brien PJ. Cellular and in vivo hepatotoxicity caused by green tea and phenolic acids and catechins. Free Rad Biol Med. 2006;40:570-580.

43. Nassiri-Asl M, Hosseinzadeh H. Review of the pharmacological effects of Vitis vinifera (grape) and its bioactive compounds. Phytother Res. 2009;23:1197-1204.

44. Duthie GG, Duthie SJ, Kyle JAM. Plant polyphenols in cancer and heart disease: implications as nutritional antioxidants. Nutr Res Rev. 2000;13:79-106.

45. Andrade RG, Ginani JS, Lopes GK, Dutra F, Alonso A, HermesLima M. Tannic acid inhibits in vitro iron-dependent free radical formation. Biochimie. 2006;88:1287-1296.

46. Lopes GK, HM, Hermes-Lima M. Polyphenol tannic acid inhibits hydroxyl radical formation from Fenton reaction by complexing ferrous ions. Biochim Biophys Acta. 1999;1472:142-152.

47. Lambert JD, Sang S, Yang CS. Possible controversy over dietary polyphenols: Benefits vs risks. Chem Res Toxicol. 2007;20:583-585.

48. Halliwell B. Are polyphenols antioxidants or pro-oxidants? What do we learn from cell culture and in vivo studies? Arch Biochem Biophys. 2008;476:107-112.

49. Lambert JD, Kwon SJ, Hong J, Yang JS. Salivary hydrogen peroxide produced by holding or chewing green tea in the oral cavity. Free Radic Res. 2007;41:850-853.

50. Weisburg JH, Weissman DB, Sedaghat T, Babich T. In vitro cytotoxicity of epigallocatechin gallate and tea extracts to cancerous and normal cells from the human oral cavity. Basic Clin Pharmacol Toxicol. 2004;95:191-200.

51. Hong J, Lu H, Meng X, Ryu JH, Hara Y, Yang CS. Stability, cellular uptake, biotransformation, and efflux of tea polyphenol (-)-epigallocatechin-3gallate in HT-29 human colon adenocarcinoma cells. Cancer Res. 2002;62:7241-7246.

52. Hou Z, Sang S, You H, et al. Mechanism of action of (-)-epigallocatechin3-gallate: Auto-oxidation-dependent inactivation of epiderma growth factor receptor and direct effects on growth inhibition in human esophageal cancer KYSE 150 cells. Cancer Res. 2005;65: 8049-8056.

53. Messina M, Loprinzi C. Soy for breast cancer survivors: A critical review of the literature. J Nutr. 2001;131:3095S-3108S

54. Messina MJ, Wood CL. Soy isoflavones, estrogen therapy, and breast cancer risk: Analysis and commentary. Nutrition J. 2008;7:17.

55. Barnes S. Soy isoflavones-Phytoestrogens and what else? J Nutr. 2004;134:1225S-1228S

56. Nichenametla SN, Taruscio TG, Barney DL, Exan JH. A review of the effects and mechanisms of polyphenolics in cancer. Crit Rev Food Sci Nutr. 2006;46:161-183.

57. Hsieh CY, Santell RC, Haslam SZ, Helferich WG. Estrogenic effects of genistein on the growth of estrogen receptor-positive human breast cancer (MCF-7) cells in vitro and in vivo. Cancer Res. 2001;58: 3833-3838.

58. Allred CD, Ju HY, Allred KF, Chang J, Helferich WG. Dietary genistin stimulates growth of estrogen-dependent breast cancer tumors similar to that observed with genistein. Carcinogenesis. 2001;22:1667-1673.
59. Petrakis NL, Barnes S, King EB, Lowenstein J, Wiencke J, Lee MM. Stimulatory influence of soy protein isolate on breast secretion in pre- and postmenopausal women. Cancer Epidemiol Biomarkers Prev. 1996;5:785-794

60. Hargreaves DF, Potten CS, Harding C, Shaw LE, Morton MM, Roberts SA. Two-week dietary soy supplementation has an estrogenic effect on normal premenopausal breast. J Clin Endocrinol Metab. 1999;84:4017-4024.

61. Ferguson LR. Role of plant polyphenols in genomic stability. Mutat Res. 2001;475:89-111.

62. Strick R, Strissel PL, Borgers S, Smith SL, Rowley JD. Dietary bioflavonoids induce cleavage in the $M L L$ gene and may contribute to infant leukemia. Proc Natl Acad Sci U S A. 2000;97:4790-4795.

63. Parr AJ, Bolwell GP. Phenols in the plant and in man. The potential for possible nutritional enhancement of the diet by modifying the phenols content or profile. J Sci Food Agric. 2000;80:985-1012.

64. Tanaka T, Kojima T, Kawamori T, et al. Inhibition of 4-nitroquinoline1-oxide-induced rat tongue carcinogenesis by the naturally occurring plant phenolics caffeic, ellagic, chlorogenic and ferulic acids. Carcinogenesis. 1993;14:1321-1325.

65. Hagiwara A, Hirose M, Takahashhi S, Ogawa K, Shirai T, Ito N. Forestomach and kidney carcinogenicity of caffeic acid in F344 rats and C57BL/6N x C3H/HeN F1 mice. Cancer Res. 1991;51:5655-5660.

66. Hirose M, Kawabe M, Shibata M, Takahashi S, Okazaki S, Ito N. Influence of caffeic acid and other o-dihydroxybenzene derivatives on N-methyl-N'-nitro-N-nitrosoguanidine-initiated rat forestomach carcinogenesis. Carcinogenesis. 1992;13:1825-1828.

67. Isbrucker RA, Edwards JA, Wolz E, Davidovich A, Bausch J. Safety studies on epigallocatechin gallate (EGCG) preparations. Part 2 : Dermal, acute and short-term toxicity studies. Food Chem Toxicol. 2005;44:636-650.

68. Cerda B, Ceron JJ, Tomas-barberan FA, Espin JC. Repeated oral administration of high doses of the pomegranate ellagitannin punicalagin to rats for 37 days is not toxic. J Agric Food Chem. 2003;51:3493-3501.

69. Ray S, Bagchi D, Lim PM. Acute and long-term safety evaluation of a novel IH636 grape seed proanthocyanidin extract. Res Comm Mol Pathol Pharmacol. 2001;109:165-197.

70. Dunnick JK, Hailey JR. Toxicity and carcinogenicity studies of quercetin, a natural component of foods. Fundam Appl Toxicol. 1992;19:423-431.

71. Zhu BT, Liehr JG. Inhibition of O-methyltransferase-catalyzed O-methylation of 2 and 4-hydroxyestradiol by quercetin: possible role in estradiol-induced tumorigenesis in hamster kidney. J Biol Chem. 1996;271:1357-1363.

72. Bonkovsky HL. Hepatotoxicity associated with supplements containing Chinese green tea (Camellia sinensis). Ann Intern Med. 2006;144: 68-71.

73. Young JJF, Dragsted LO, Haraldsdottir J, et al. Green tea extract affects markers of oxidative stress postprandially: lasting antioxidant effect of a flavonoid-free diet. Br J Nutr. 2002;87:343-355.

74. Halliwell B. Establishing the significance and optimal intake of dietary antioxidants: the biomarker concept. Nutr Rev. 1999;57:104-113.

75. Perron NR, Brumaghim JL. A review of the antioxidant mechanisms of polyphenol compounds related to iron binding. Cell Biochem Biophys. 2009;53:75-100.

76. Olthof MR, Hollman PC, Zock PL, Katan MB. Consumption of high doses of chlorogenic acid, present in coffee, or of black tea increases plasma total homocysteine concentrations in humans. Am J Clin Nutr. 2001;73:532-538.

77. Hodgson JM, Burke V, Beilin LJ, Croft KD, Puddey IB. Can black tea influence plasma total homocysteine concentrations? Am J Clin Nutr. 2003;77:907-911.

78. Kiani J, Imam S. Medicinal importance of grapefruit juice and its interaction with various drugs. Nutr J. 2007;6:33

79. Dahan A, Altman H. Food-drug interaction: Grapefruit juice augments drug bioavailability-mechanism, extent, and relevance. Eur J Clin Nutr. 2004;58:1-9. 
80. Williams D, Feely J. Pharmacokinetic-pharmacodynamic drug interactions with HMG-CoA reductase inhibitors. Clin Pharmacokinet. 2002;41:343-370.

81. Schmiedlin-Ren P, Edwards DJ, Fitzsimmons ME, et al. Mechanisms of enhanced oral availability of CYP3A4 substrates by grapefruit constituents. Drug Metab Dispos. 1997;25:1228-1233.

82. Fuhr U, Kummert AL. The fate of naringin in humans: a key to grapefruit juice-drug interactions? Clin Pharmacol Ther. 1995;58:365-373.

83. Hare JT, Elliott DP. Grapefruit juice and potential drug interactions. Consult Pharm. 2003;18:466-472.
84. Galati G, Teng S, Moridani MY, Chan TS, O’Brien J. Cancer chemoprevention and apoptosis mechanisms induced by dietary polyphenolics. Drug Metab Drug Int. 2000;17:311-349.

85. Schilter B, Andersson C, Anton R, et al. Guidance for the safety assessment of botanicals and botanical preparations for use in food and food supplements. Food Chem Toxicol. 2003;41:1625-1649.

86. Spencer JP, Abd El Mohsen MM, Minihane AM, Mathers JC. Biomarkers of the intake of dietary polyphenols: strengths, limitations and application in nutrition. Br J Nutr. 2008;99:12-22.

\section{Publish your work in this journal}

Nutrition and Dietary Supplements is an international, peer-reviewed, open access journal focusing on research into nutritional requirements in health and disease, impact on metabolism and the identification and optimal use of dietary strategies and supplements necessary for normal growth and development. The journal welcomes papers covering original research, basic science, clinical \& epidemiological studies, reviews and evaluations, guidelines, expert opinion and commentary, case reports and extended reports. The manuscript management system is completely online and includes a very quick and fair peer-review system, which is all easy to use.

Submit your manuscript here: http://www.dovepress.com/nutrition-and-dietary-supplements-journal 